\title{
Flat-top temperature tuning response in periodically-poled nonlinear crystals
}

\author{
Huw E. Major, Anna C. Peacock, James C. Gates, Corin B.E. Gawith, Peter G.R. Smith \\ Optoelectronics Research Centre (ORC), University of Southampton, SO17 1BJ \\ Southampton, United Kingdom \\ Phone: $+44(0) 2380594530$ \\ Fax: +44 (0)23 80593149 \\ E mail: hem@orc.soton.ac.uk
}

\begin{abstract}
Second harmonic generation via periodically-poled nonlinear materials offers an efficient means of generating high-quality visible light that would be otherwise unattainable with traditional laser sources. While this technology has the potential for implementation in many mass-industrial applications, temperature stability requirements of 0.1 deg.C can make packaging with a pump source problematic.

Our route towards solving this issue is to mathematically model the placement of poled domains and convert the standard sinc-shaped temperature-tuning response of a uniform grating to a flat-top temperature stability across several degrees. We have achieved a rapid means of performing this process based on repeated local changes of grating layout and subsequent Bloombergen-style analysis of the second harmonic, successive iterations of which quickly lead to the desired temperature tuning profile.

Using our high fidelity poling technique we have achieved precise placement of poled domains in Lithium Niobate based on the resulting mathematical models. These initial devices provide more than 4 deg.C flat-top temperature stability, albeit with a corresponding loss in operational efficiency. Our aim is to implement improved designs in magnesium-doped Lithium Niobate for packaging with near-room temperature diode-based pump sources, as could be applied towards RGB TV and projector applications.
\end{abstract}

Keywords: Nonlinear, Poled, QPM, Temperature Stable, Bloombergen

Principal author biography: Huw Major is working towards a doctorate in quantum optic sources at the Optoelectronics Research Centre (Southampton). He holds a Bachelor in Electronic Engineering from the University of Southampton. 